ARTICLE

Received 21 Aug 2015 | Accepted 27 Jan 2016 | Published 1 Mar 2016

DOl: $10.1038 /$ ncomms10852

OPEN

\title{
Observation of a three-dimensional quasi-long-range electronic supermodulation in $\mathrm{YBa}_{2} \mathrm{Cu}_{3} \mathrm{O}_{7-x} / \mathrm{La}_{0.7} \mathrm{Ca}_{0.3} \mathrm{MnO}_{3}$ heterostructures
}

Junfeng He ${ }^{1, \star}$, Padraic Shafer ${ }^{2, \star}$, Thomas R. Mion ${ }^{1, \star}, \mathrm{Vu}$ Thanh Tra ${ }^{3}$, Qing He ${ }^{4}$, J. Kong ${ }^{1}$, Y.-D. Chuang ${ }^{2}$, W.L. Yang ${ }^{2}$, M.J. Graf', J.-Y. Lin ${ }^{2,3}$, Y.-H. Chu ${ }^{5,6}$, E. Arenholz ${ }^{2} \&$ Rui-Hua He ${ }^{1}$

Recent developments in high-temperature superconductivity highlight a generic tendency of the cuprates to develop competing electronic (charge) supermodulations. While coupled with the lattice and showing different characteristics in different materials, these supermodulations themselves are generally conceived to be quasi-two-dimensional, residing mainly in individual $\mathrm{CuO}_{2}$ planes, and poorly correlated along the $\mathrm{c}$ axis. Here we observed with resonant elastic $\mathrm{X}$-ray scattering a distinct type of electronic supermodulation in $\mathrm{YBa}_{2} \mathrm{Cu}_{3} \mathrm{O}_{7-x}$ (YBCO) thin films grown epitaxially on $\mathrm{La}_{0.7} \mathrm{Ca}_{0.3} \mathrm{MnO}_{3}$ (LCMO). This supermodulation has a periodicity nearly commensurate with four lattice constants in-plane, eight out of plane, with long correlation lengths in three dimensions. It sets in far above the superconducting transition temperature and competes with superconductivity below this temperature for electronic states predominantly in the $\mathrm{CuO}_{2}$ plane. Our finding sheds light on the nature of charge ordering in cuprates as well as a reported long-range proximity effect between superconductivity and ferromagnetism in YBCO/LCMO heterostructures.

\footnotetext{
${ }^{1}$ Department of Physics, Boston College, Chestnut Hill, Massachusetts 02467, USA. ${ }^{2}$ Advanced Light Source, Lawrence Berkeley National Laboratory, Berkeley, California 94720, USA. ${ }^{3}$ Institute of Physics, National Chiao Tung University, Hsinchu 30010, Taiwan. ${ }^{4}$ Department of Physics, Durham University, Durham DH13LE, UK. ${ }^{5}$ Institute of Physics, Academia Sinica, Taipei 11529, Taiwan. ${ }^{6}$ Department of Materials Science and Engineering, National Chiao Tung University, Hsinchu 30010, Taiwan. * These authors contributed equally to this work. Correspondence and requests for materials should be addressed to R.-H.H. (email: ruihua.he@bc.edu).
} 
key feature of unconventional superconductors is the presence of multiple ordering tendencies, with some competing (and often coexisting) with superconductivity for electronic states otherwise available for pairing. A case quite often encountered is that these competing orders are affected by, if not entirely driven by, the same underlying interactions that mediate superconductivity ${ }^{1}$. Elucidating the exact nature of these interactions would thus promise both useful practical guidance to manipulate superconductivity (via effects on the competing orders) and complementary theoretical insights into the microscopic mechanisms for superconductivity.

Remarkable progress has been made in recent years in identifying charge ordering as a generic competing instability within the cuprate superconductors; nevertheless, a unified understanding of its diverse manifestations in different materials is still lacking. In general, the observed charge orders fall into two main categories. One has an in-plane periodicity close to four lattice constants (period-4) and has been found in La-based cuprates ${ }^{2-4}, \quad \mathrm{Ca}_{2}-{ }_{x} \mathrm{Na}_{x} \mathrm{CuO}_{2} \mathrm{Cl}_{2}$ (ref. 5), $\mathrm{Bi}_{2} \mathrm{Sr}_{2} \mathrm{CaCu}_{2} \mathrm{O}_{8+\delta}$ (Bi2212) (refs 6-8), $\mathrm{YBCO}^{9}, \mathrm{Bi}_{2} \mathrm{Sr}_{2-}{ }_{x} \mathrm{La}_{x} \mathrm{CuO}_{6+\delta}$ (Bi2201) (ref. 10) and $\mathrm{Nd}_{2}{ }_{x} \mathrm{Ce}_{x} \mathrm{CuO}_{4}$ (ref. 11). Another category has a periodicity generally different from four lattice constants that varies in $\mathrm{Bi} 2201$ (ref. 12), $\mathrm{YBCO}^{13-17}, \mathrm{Bi} 2212$ (ref. 8) and $\mathrm{HgBa}_{2} \mathrm{CuO}_{4+\delta}$ (ref. 18). For bulk YBCO, charge order with periodicity close to three lattice constants (period-3) was observed in resonant elastic X-ray scattering (REXS) and hard X-ray diffraction experiments in zero or relatively low magnetic field. This ordering has an onset at $\sim 110-160 \mathrm{~K}$ upon cooling and maintains short-range order at even lower temperatures ${ }^{13-17}$. A period-4 charge order was found in NMR experiments at high fields, which has long-range order and develops below $\sim 45-60 \mathrm{~K}$ in addition to the period- 3 order ${ }^{19}$. It remains uncertain whether the two categories of order reflect the same charge ordering mechanism in the cuprates that is prone to the effects of various internal (crystalline environment, quenched disorder) and external factors (magnetic field) or whether they should be attributed to distinct tendencies of different origins, each competing with superconductivity ${ }^{20}$.

In this paper, we report the observation of a distinct electronic order in YBCO thin films grown epitaxially on LCMO layers. This order shares similarities with both categories of charge order found in bulk YBCO, yet shows important distinctions. Like the bulk orders, the electronic order propagates along the $\mathrm{Cu}-\mathrm{O}$ bond directions, predominantly involves electronic states in the $\mathrm{CuO}_{2}$ plane, and competes with superconductivity. It has a similar periodicity as the bulk period-4 charge order and also shares a long correlation length in the $\mathrm{CuO}_{2}$ plane, but has a much higher onset temperature, $\sim 220 \mathrm{~K}$. These characteristics are different from those of the period- 3 bulk charge orders found under similar experimental conditions using the same technique, REXS. Crucially, this electronic order exhibits a remarkable correlation along the crystalline $c$ axis, which is unprecedented among all the charge orders found so far in the cuprates. We found that the onset of the electronic order does not coincide with that of bulk ferromagnetism in the LCMO layer. Our observations add a significant piece to the puzzle of high-temperature superconductivity, providing insights into the nature of charge ordering in cuprates. In addition, this three-dimensional (3D) long-range competing order in YBCO/LCMO heterostructures provides an angle for interpreting the long-range proximity effect between superconductivity and ferromagnetism reported in this system.

\section{Results}

In-plane component of the electronic supermodulation. REXS combines the periodic spatial information of X-ray diffraction with the sensitivity to local electronic bonding environment found in soft X-ray absorption fine structure. Using photons with energy near the $\mathrm{Cu} L_{3}$ absorption edge $\left(2 p_{3 / 2} \rightarrow 3 d\right.$ transition; Fig. 1b), REXS can directly detect the ordering of valence electronic states in cuprates that involves modulations in their charge density and/or band energy. Earlier experiments have shown that REXS is particularly sensitive to charge order ${ }^{13,15,16}$.

The YBCO thin films were grown epitaxially on LCMO layers (Supplementary Fig. 1). The intensity of X-rays scattered from YBCO/LCMO films (Fig. 1a) was measured as a function of momentum transfer along primary crystallographic directions using photons both on resonance $(930.85 \mathrm{eV})$ and off resonance $(926 \mathrm{eV})$. Diffraction spots (Fig. 1c) were observed with resonant photons at symmetric points in reciprocal space, $( \pm H, 0, L)$, where indices $H$ and $L$ indicate scaling of the reciprocal lattice units, $2 \pi / a$ and $2 \pi / c$, respectively. Line cuts through the diffraction peaks along the in-plane $\mathrm{Cu}-\mathrm{O}$ bond direction $(a$ axis) are shown for $(0.245,0,1.38)$ in Fig. $1 \mathrm{~d}$ and $(-0.245$, $0,1.38)$ in Fig. 1e. The on-resonance diffraction peaks are superimposed upon a diffuse background of comparable intensity. The diffuse background contains components of X-ray fluorescence from the sample, as well as scattered X-rays that do not contribute to the diffraction signal. As shown in Fig. 1d,e, the on-resonance diffuse background-measured at detector angles slightly away from the diffraction condition-closely matches the shape of the scattered intensity line cuts everywhere except in close proximity to $H= \pm 0.245$. By subtracting the diffuse background, the true on-resonance diffraction peaks are revealed (also shown in Fig. 1d,e).

These diffraction peaks are located at $|H|=0.245 \pm 0.005$ (the error bars come from the uncertainties in the determination of the peak positions), very close to the commensurate wavevector $1 / 4$, indicative of a supermodulation having a period close to $4 a(a=3.855 \AA$ (ref. 13)) along the $\mathrm{Cu}-\mathrm{O}$ bond direction. This periodicity is different from those of the period-3 charge orders found in the bulk YBCO by REXS with wavevector $\sim 0.31-0.34$ (refs 13-17), but consistent with the one inferred from a NMR study 9 . The observed diffraction peak has a full width half maximum $\delta H=0.0075$, corresponding to a correlation length $\xi_{a b} \doteq a(\pi \delta H)^{-1} \sim 164 \AA(\sim 42$ unit cells, u.c. $)$ for the supermodulation, which is two times larger than the longest correlation length reported on the period-3 charge orders in bulk $\mathrm{YBCO}^{16,17}$. We note that both the periodicity and correlation length of the supermodulation in YBCO/LCMO are quantitatively similar to those of the long-range charge stripe order in $\mathrm{La}_{1.875} \mathrm{Ba}_{0.125} \mathrm{CuO}_{4}$ (ref. 21; Fig. 1f). Moreover, we observed that the supermodulation diffraction peaks diminish rapidly away from resonance (Fig. 1f). Such a strong resonant effect indicates a sizable electronic character of the supermodulation that necessarily involves $\mathrm{Cu} 3 d$ valence states.

Out-of-plane component of the electronic supermodulation. Figure 2a shows the scattered intensity and nearby background intensity as a function of $L$ at $(-0.245,0, L)$. Their difference is plotted in Fig. $2 b$ to reveal the true diffraction peak, similar to the line cuts along $H$ shown in Fig.1. The diffracted intensity exhibits a strong $L$ dependence, dominated by a prominent peak with full width half maximum $\delta L=0.017$. This suggests a remarkable correlation of the supermodulation along the $c$ axis with a correlation length $\xi_{c} \doteq c(\pi \delta L)^{-1} \sim 219 \AA(\sim 19$ u.c.; $c=11.64 \AA)$. This result strongly contrasts with the weak $L$-dependent modulations of the diffracted intensity and the short $c$ axis correlation length $(\sim 1$ u.c.) of the charge orders found in various bulk cuprates by REXS $4,10,13$. It points to a $3 \mathrm{D}$ quasi-long-range order of the electronic supermodulation in YBCO/LCMO. 
The diffraction peak is located at $L=1.38 \pm 0.02$, very close to the commensurate wavevector $L=11 / 8$, hinting at an approximate $8 c$ period of the supermodulation along the $c$ axis. Within the experimentally accessible $L$ range, no diffraction peak is found near commensurate wavevectors $L=10 / 8$ and $L=12 / 8$. As shown in Supplementary Fig. 2, we have calculated diffraction structure factors for four representative types of period- 8 charge density modulation along the $c$ axis each with a different phase relation within the $\mathrm{CuO}_{2}$ bilayer as well as between neighbouring bilayers. A common feature of these calculations is that diffraction peak is present at odd harmonics of $L=1 / 8$ but not at even harmonics, although the relative peak intensity varies with the form of modulation. Such a robust qualitative aspect of the calculations is consistent with our experimental observation for the out-of-plane component of the electronic supermodulation (cf. Fig. 2b,c).

Temperature evolution of the electronic supermodulation. We have studied the diffraction peak centred at $(-0.245,0,1.38)$ as a function of temperature on the same YBCO/LCMO sample, with results summarized in Fig. 3a,b. No diffraction peak is found at room temperature. It emerges upon lowering the temperature, showing a clear onset behaviour that appears more abruptly than those observed on the period-3 charge orders in bulk $\mathrm{YBCO}^{13-17}$. This onset occurs at $T_{\text {co }} \sim 220 \mathrm{~K}$, substantially higher than the period-3 charge ordering onset $\sim 150 \mathrm{~K}$. $T_{\text {co }}$ is also significantly higher than the Curie temperature of the LCMO layer $T_{\mathrm{m}} \sim 175 \mathrm{~K}$, which was determined by a magnetization measurement on the YBCO/LCMO sample (the red curve in the inset of Fig. 3b).

The diffracted intensity grows at first rapidly upon further lowering the temperature and then more gradually $\sim 140 \mathrm{~K}$ (Fig. 3b). The intensity reaches a maximum at $T_{\max } \sim 50 \mathrm{~K}$, below which intensity decreases. During the entire temperature evolution the diffraction peak stays at roughly the same position (Fig. 3a) with only modest changes to the peak width (Supplementary Fig. 3). We note that the superconducting transition temperature of the YBCO layer $T_{\mathrm{c}}$ was determined from a four-probe resistivity measurement on the YBCO/LCMO sample to be $55 \pm 10 \mathrm{~K}$ (the blue curve in the inset of Fig. 3b), which is nearly indistinguishable from $T_{\max }$ on this sample (sample \#1).

We have performed similar measurements on a second YBCO/LCMO sample (sample \#2) that was grown with a different interface termination than sample \#1 (see Methods and Discussion). Sample \#2 shows a slightly higher $T_{\mathrm{c}}=72 \pm 7 \mathrm{~K}$
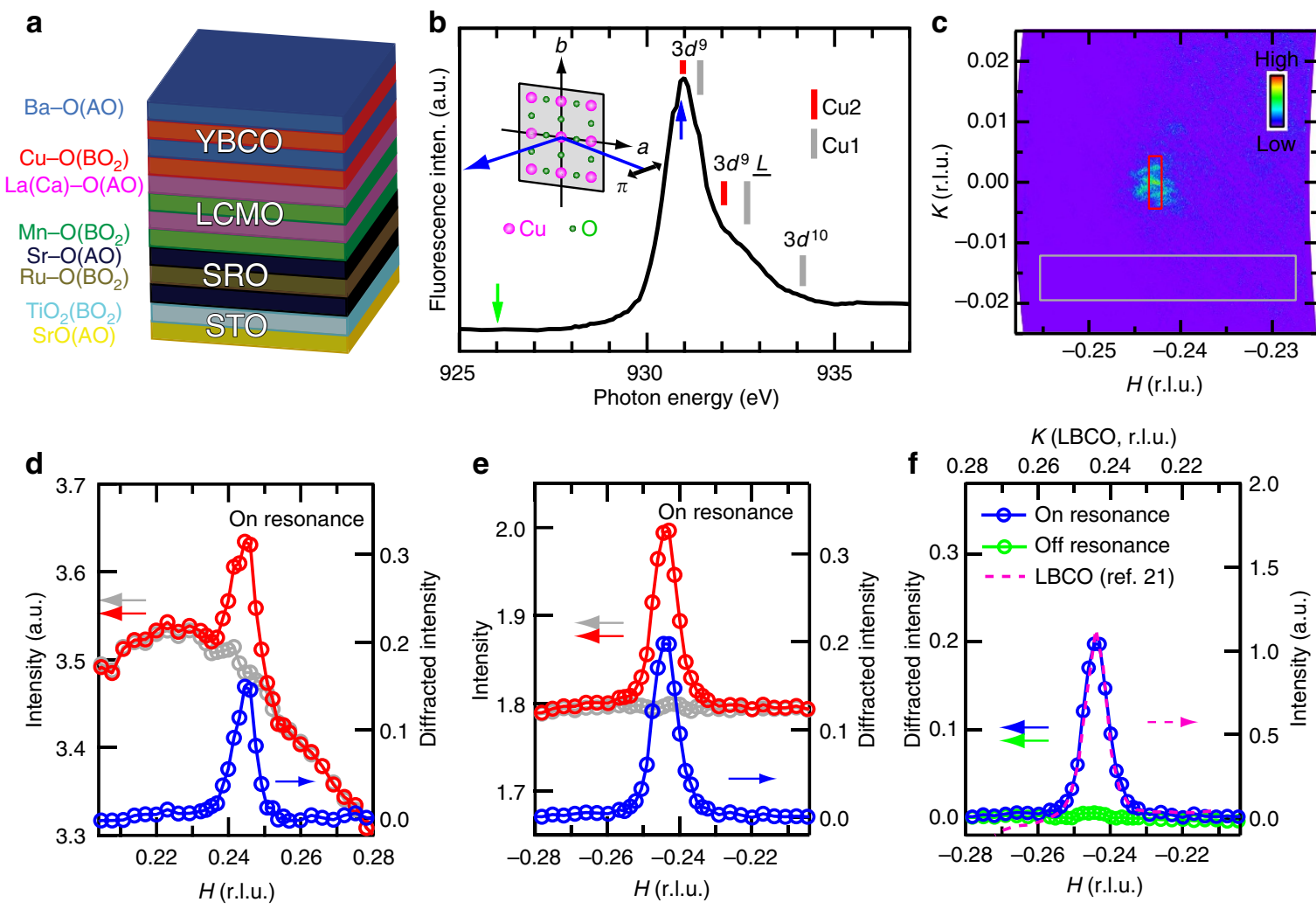

Figure 1 | In-plane component of the electronic supermodulation. (a) Schematic of the heterostructure in sample \#1. The individual atomic layers $\left(\mathrm{AO}\right.$ and $\mathrm{BO}_{2}$ ) of the $\mathrm{ABO}_{3}$-type perovskite are indicated by different colours. (b) X-ray absorption spectrum measured near the $\mathrm{Cu} L_{3}$ absorption edge in total fluorescence yield. Main configuration-dependent contributions to the spectrum are marked by red and grey bars as reproduced from ref. 13. Red and grey bars mark the energy positions of states derived from the $\mathrm{Cu}$ sites in the $\mathrm{CuO}_{2}$ plane (Cu2) and those in the $\mathrm{Cu}-\mathrm{O}$ chain layer (Cu1), respectively. Inset: schematic of the scattering geometry with linear $(\pi)$ incident polarization in the horizontal scattering plane. (c) In-plane reciprocal space projection derived from a single CCD image measured at $930.85 \mathrm{eV}$ (indicated by blue arrow in $\mathbf{b}$ ) showing a diffraction peak near $(-0.245,0,1.38)$. Note that the raw image contains the $L$ range from $\sim 1.31$ to $\sim 1.45$, and only its projection onto the $(H, K, 0)$ plane is shown here. (d,e) Scattered intensity line cuts along $H$ measured at $50 \mathrm{~K}$ and $930.85 \mathrm{eV}$ at fixed $L=1.38$ and $K=0$. Each point on the red (grey) curve indicates average intensity in the region of the corresponding CCD image marked by the red (grey) rectangle in $\mathbf{c}$. Blue curve is the difference between red and grey curves showing the diffracted intensity. The intensity in $\mathbf{e}$ and diffracted intensity in $\mathbf{d - f}$ all share the same scale with the intensity in $\mathbf{d}$. (f) Diffracted intensity measured at $926 \mathrm{eV}$ (green curve, indicated by the green arrow in $\mathbf{b}$ ) and $930.85 \mathrm{eV}$ (blue curve, reproduced from $\mathbf{e}$ ) at fixed $L=1.38$ and $K=0$, compared alongside the $K$ scan of $\mathrm{La}_{1.875} \mathrm{Ba}_{0.125} \mathrm{CuO}_{4}$ (reproduced from ref. 21; note that equivalent modulations exist along both the $H$ and $K$ directions in the low-temperature tetragonal phase of $\mathrm{La}_{1.875} \mathrm{Ba}_{0.125} \mathrm{CuO}_{4}$ ). All results were obtained from sample \#1. r.l.u., reciprocal lattice units. 
than sample \# 1 but a similar $T_{\mathrm{m}}$ (the inset of Fig. $3 \mathrm{~d}$ ). A similar supermodulation was found in this sample, with an overall similar temperature evolution of the diffraction peak (cf. Fig. 3a,b versus Fig. $3 c, d$ ). A notable difference is $T_{\text {max }}$, which is now $\sim 75 \mathrm{~K}$. We emphasize that $T_{\max } \approx T_{\mathrm{c}}$ in each sample.

The observed suppression of the diffraction peak below the superconducting transition in $\mathrm{YBCO} / \mathrm{LCMO}$ is reminiscent of those observed in bulk $\mathrm{YBCO}^{13-17}$. Similar temperaturedependent behaviour has generally been taken as evidence for a microscopic coexistence and dynamic competition between two distinct orders ${ }^{22,23}$. Such coexistence can occur in a homogenous state. Nevertheless, a rather typical situation is that the two orders are phase separated at the nanoscale with a volume fraction that changes with temperature. Regardless of which type of spatial coexistence is present, the observed temperature dependence suggests that a considerable portion of electronic states that participated in the electronic supermodulation above $T_{c}$ are instead consumed by superconductivity below $T_{c}$. Because the states involved in superconductivity lie primarily within the $\mathrm{CuO}_{2}$ plane, the electronic supermodulation in $\mathrm{YBCO} / \mathrm{LCMO}$ is accordingly expected to have a sizable contribution from the states in the $\mathrm{CuO}_{2}$ plane.

Primary $\mathrm{CuO}_{2}$ plane origin of the electronic supermodulation. A period- 4 supermodulation in the $a b$ plane could in principle be caused by the ordering of oxygen into arrays of full and empty chains in the $\mathrm{Cu}-\mathrm{O}$ chain layer. While such period-4 (aka ortho-IV) oxygen order has, to our knowledge, not been reported in YBCO thin films, it has been observed in bulk YBCO at and above the optimal doping level ${ }^{24,25}$. Like other oxygen orderings, the ortho-IV order persists well above room temperature (up to $500 \mathrm{~K}$ ) and has a weak temperature dependence, with no anomaly observed at $T_{\mathrm{c}}$. It remains short-ranged in the $a b$ plane and poorly correlated along the $c$ axis, with correlation lengths $\sim 3-6$ and $<1$ u.c., respectively. All of these characteristics strongly contrast with our observations in YBCO/LCMO.

Our REXS experiment provides further insights into the chain $(\mathrm{Cu} 1)$ versus plane $(\mathrm{Cu} 2)$ origin of the observed electronic supermodulation; here we maintain the copper site ( $\mathrm{Cul}$ and $\mathrm{Cu} 2$ ) terminology used in ref. 13. By analysing the photon energy dependence of the diffracted intensity, we directly probe the site, configuration and orbital nature of the states that participate in the associated supermodulation. This resonance profile of the diffraction peak is generally distinct from the X-ray absorption spectrum that is obtained, for example, from the fluorescence near the $\mathrm{Cu} L_{3}$ edge that involves all unoccupied $\mathrm{Cu} 3 d$ orbitals, regardless of whether they are ordered. As shown in Fig. 4a, the resonance profile measured at $(-0.245,0,1.38)$ in our YBCO/LCMO sample shows a dominant peak close to the $L_{3}$ absorption maximum at $931 \mathrm{eV}$. This situation qualitatively resembles the case of the period-3 charge order in bulk YBCO, an example of which is reproduced from the literature in Fig. 4b (ref. 15). This peak has been ascribed to a $3 d^{9}$ ground-state configuration at the $\mathrm{Cu}(\mathrm{Cu} 2)$ site in the $\mathrm{CuO}_{2}$ plane (as marked in Fig. 1b) (refs 13,15). In our study, the peak is observed $\sim 0.55 \mathrm{eV}$ below the absorption maximum. This finite energy difference between the $\mathrm{Cu} 23 d^{9}$ peak of the resonance profile and the absorption maximum is not uncommon for cuprates, and its size has been found to vary considerably among different experiments $3,4,15,26$ even in the same class of materials. We note that the possible presence of 'dead' layers on the sample surface could account for this energy difference. These surface layers would not contribute to the scattered intensity but would absorb and effectively suppress the peak intensity around the absorption maximum, thereby shifting the apparent diffraction peak towards lower energy. On the other hand, if this energy difference is intrinsic, it may provide a useful constraint on the type of spatial modulation that underlies the electronic supermodulation, for example, whether it is dominated by direct modulation of the local charge density or related properties, such as the local $\mathrm{Cu} 2 p \rightarrow 3 d$ transition energy ${ }^{15}$. In this context, future studies might investigate to what extent the larger energy difference found in YBCO/LCMO versus in the bulk (cf. Fig. 4a versus Fig. 4b) reflects the characteristics of these distinct electronic structure modulations.

We next consider that a shoulder is clearly present in our resonance profile at $\sim 1 \mathrm{eV}$ above the main peak (Fig. $4 \mathrm{a}$ ). On the basis of this energy separation, we tentatively assign the shoulder feature to the $3 d^{9} L$ configuration at the $\mathrm{Cu} 2$ site (as marked in Fig. 1b) (ref. 13). Nevertheless, a theoretical calculation that takes into account all possible states in proximity, such as the $3 d^{9}$ configuration at the $\mathrm{Cu}(\mathrm{Cu} 1)$ site in the $\mathrm{Cu}-\mathrm{O}$ chain layer, might be needed for a concrete understanding of this secondary feature. Most notably the well-defined three dimensionality of the electronic supermodulation probably involves cooperation with the entire lattice, which would necessitate that additional sites outside of the $\mathrm{CuO}_{2}$ planes contribute to the resonance profile. Its detailed line shape thus holds a key to further elucidate the electronic supermodulation and its distinction from the bulk charge order.

In contrast, our measured resonance profile looks qualitatively different from those for various oxygen orders in the $\mathrm{Cu}-\mathrm{O}$ chain layer of bulk YBCO, which we reproduce in Fig. 4c (refs 15,27).
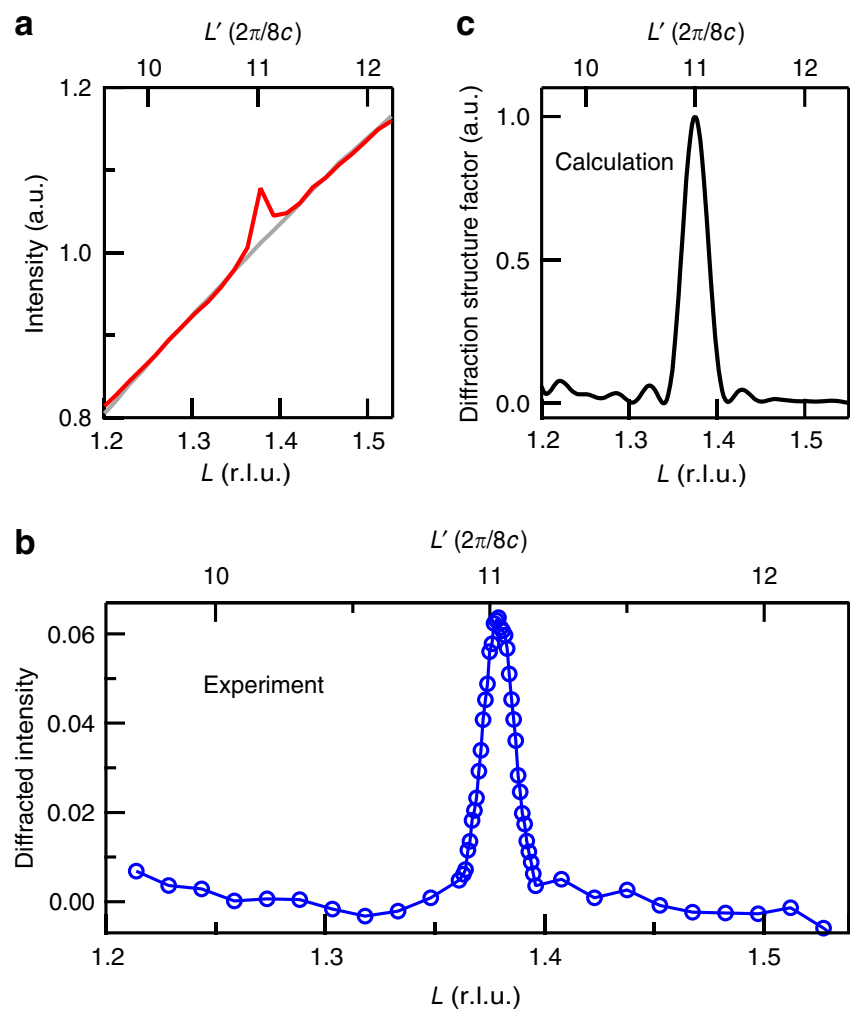

Figure 2 | Out-of-plane component of the electronic supermodulation.

(a) Scattered intensity line cuts along $L$ measured on sample \#1 at $50 \mathrm{~K}$ and $930.6 \mathrm{eV}$ at fixed $H=-0.245$ and $K=0$. Average intensity in the peak region and background region of the CCD image are shown in red and grey curves, respectively. (b) Diffracted intensity for the same line cut, which is the difference between red and grey curves in $\mathbf{a}$. The diffracted intensity in $\mathbf{b}$ shares the same scale with the intensity in a. (c) Calculated diffraction structure factor for one representative type of charge density modulation along the $c$ axis. r.l.u., reciprocal lattice units. 

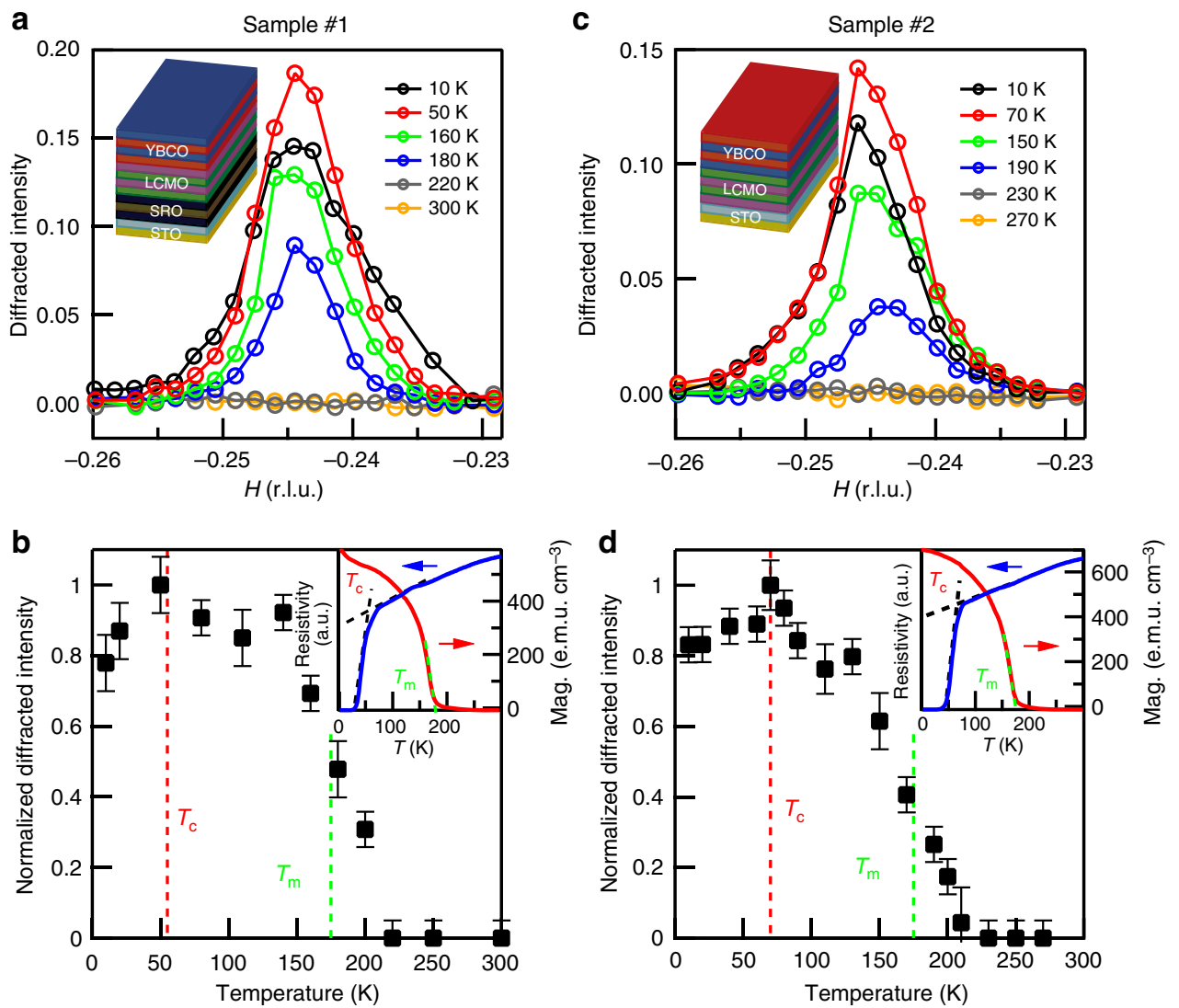

Figure 3 | Temperature evolution of the electronic supermodulation. (a) Diffracted intensity of a line cut along $\mathrm{H}$ measured at resonance (930.85 eV) on sample \#1 at selected temperatures at fixed $L=1.38$ and $K=0$. (b) Temperature dependence of the peak intensity (normalized to the maximum value) in a. Red (green) dashed line marks $T_{\mathrm{c}}\left(T_{\mathrm{m}}\right)$ determined from the resistivity (magnetization) measurement shown in the insets. (c,d) Results of sample \#2. The diffracted intensity in $\mathbf{a}$ and $\mathbf{c}$ share the same scale as before. $(\mathbf{a}, \mathbf{c})$ Inset: schematics of the heterostructure for samples \#1 and \#2, respectively. Error bars reflect the uncertainty due to mean-square deviation in determining the diffraction peak intensity based on curve fitting. r.l.u., reciprocal lattice units.
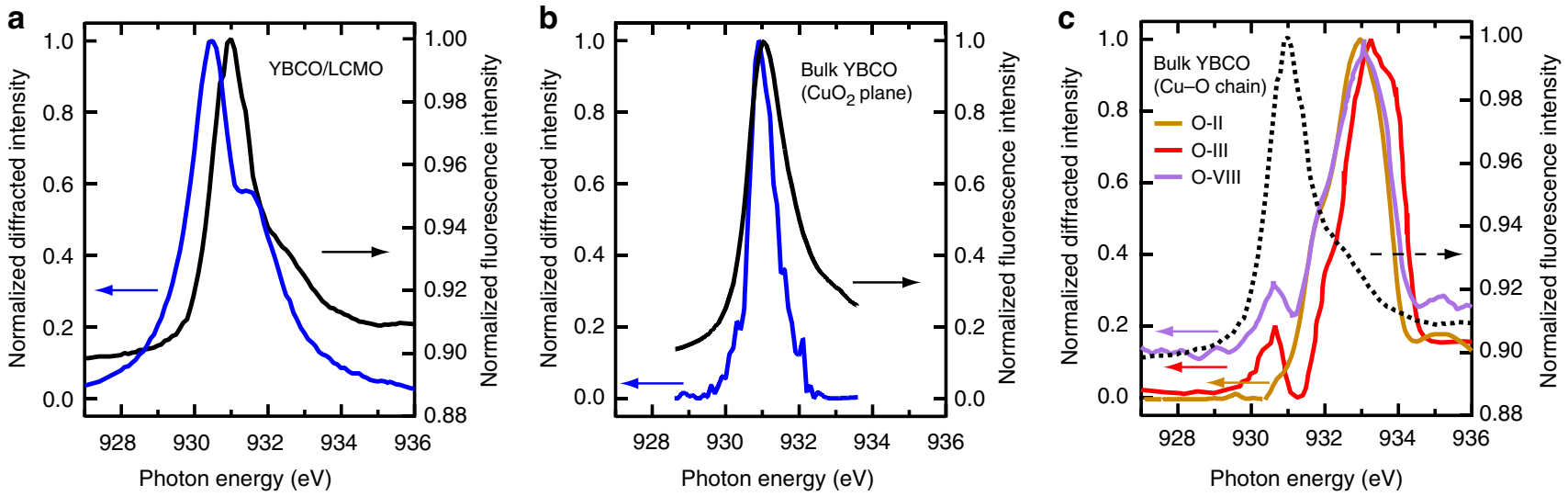

Figure 4 | Comparison on the resonance profile for various orders. (a) Photon energy dependence of the diffracted intensity (normalized to the maximum) at $(-0.245,0,1.38)$ associated with the electronic supermodulation (resonance profile; blue curve), in comparison with that of the fluorescence (X-ray absorption spectrum; black), measured on sample \#1 at $50 \mathrm{~K}$. (b) Resonance profile of the period-3 charge order and X-ray absorption spectrum in a bulk YBCO sample, reproduced from ref. 15. (c) Resonance profiles of various oxygen orderings, ortho-II, ortho-III and ortho-VIII, in different bulk YBCO samples, reproduced from refs 15,27. Dashed curve is the X-ray absorption spectrum of sample \#1 reproduced as a guide to the eye. Slight energy offsets in the energy axis between different experiments have been corrected by setting the corresponding $L_{3}$ absorption peak to the same energy. Note that conditions may vary in different experiments.

Although the spectral line shape depends on the actual configuration of the oxygen order and the doping level, a common characteristic of these resonance profiles is that the dominant peak is located at least $2 \mathrm{eV}$ above the absorption edge. The relevant states in this energy range are mostly associated with a $d^{9} L$ configuration of the Cul site ${ }^{13}$, which is directly impacted by the oxygen ordering. Because these features are so distinct from the characteristics we observed, it appears unlikely that the resonance profile of the electronic supermodulation can be consistent with an ordering of oxygen in the chain layer. 
Consistent with the temperature dependence results, all of these details gleaned from the resonance profile support a scenario in which the $\mathrm{CuO}_{2}$ planes are the primary locations of the electronic supermodulation in YBCO/LCMO.

\section{Discussion}

What is the nature of the observed electronic supermodulation? Although some unconventional orders have been proposed to exist in cuprates, only two-dimensional (2D) charge and spin orders have been found experimentally, with the in-plane charge ordering wavevectors located around $(0,0)$ and the spin ordering wavevectors around $(1 / 2,1 / 2)$. In this context, our electronic supermodulation with an in-plane wavevector $(0.245,0)$ is likely a charge order. Our 30-nm YBCO films contain structurally twinned domains and have a pseudo-tetragonal structure, similar to the case in $\mathrm{Nd}_{1.2} \mathrm{Ba}_{1.8} \mathrm{Cu}_{3} \mathrm{O}_{7}$ (NBCO) epitaxial films ${ }^{28}$. This has made it difficult to distinguish between uniaxial and biaxial supermodulations with the typical spot size $\left(100 \times 100 \mu \mathrm{m}^{2}\right)$ of our X-ray beam much larger than the domain size. Because of the relatively small photon momentum at the $\mathrm{Cu} L_{3}$ resonance, the measured intensity line cuts along $H$ and $L$ cover only a limited range in the reciprocal space (for example, $(-0.245,0,13 / 8)$ is inaccessible) and do not provide sufficient information to reconstruct the full arrangement of charge disproportionation (spatial configuration) of the supermodulation. Future scattering experiments promise further insights into this order, in terms of its charge versus spin nature by studying the polarization dependence of the diffracted intensity ${ }^{13}$; in terms of the in-plane anisotropy (order along $a$ versus $b$ axis) via spatially resolved scattering experiments; and in terms of the spatial configuration based on diffraction structure factors determined from measurements over a wider region of the reciprocal space using hard X-rays ${ }^{29}$. Despite these open questions, the 3D electronic supermodulation we directly observed is the first of its kind ever reported in cuprates.

To the extent that our observation is indeed charge order, its in-plane periodicity suggests a close link with the period-4 charge order found in bulk YBCO by NMR ${ }^{9}$. As a local probe, NMR does not readily distinguish whether an order is $2 \mathrm{D}$ or $3 \mathrm{D}$ in layered materials, although its finding on YBCO has been interpreted by assuming a $2 \mathrm{D}$ order typical for cuprates. In light of the ubiquity of period-4 charge orders in various cuprate families, it is not implausible that both orders reflect the same inherent tendency towards period-4 charge ordering. In such a scenario, possible variations in the dimensionality, correlation length and onset temperature of the orders may reflect the susceptibility of such tendency to various internal (strain, quenched disorder) and external factors (crystalline environment, magnetic field) as recently discussed ${ }^{20}$.

However, because two different types of (period-3 and period-4) electronic supermodulation have been observed in nominally the same material (YBCO) with the same technique (REXS) under similar experimental conditions, a new question is posed: what is the possible cause for such a difference? To pinpoint this cause would be the first step to understand whether or not both types of supermodulation should be attributed to distinct tendencies of different origins ${ }^{20}$.

We first consider the carrier concentration. Determining and controlling the oxygen content in oxide thin-film growth is still a general experimental challenge. While our deposition and annealing recipe is optimized for the growth of an optimally doped YBCO thin film directly on the $\mathrm{SrTiO}_{3}$ (001) substrate (Methods), the overall oxygen content of the YBCO film that is instead grown on the intermediate LCMO layer could likely shift the effective doping away from the optimal level, given the different crystalline environment during the growth. Moreover, charge transfer at the YBCO/LCMO interface tends to decrease the overall hole concentration of the YBCO layer ${ }^{30}$. It was recently found ${ }^{31}$ that the charge transfer is stronger at the $\mathrm{La}_{0.7} \mathrm{Ca}_{0.3} \mathrm{O}$-terminated interface (associated with our sample \#1 of $T_{\mathrm{c}}=55 \mathrm{~K}$ ) than at the $\mathrm{MnO}_{2}$-terminated interface (associated with our sample $\# 2$ of $T_{\mathrm{c}}=72 \mathrm{~K}$ ). It is thus likely that both the YBCO thin films in samples \#1 and \#2 with period-4 supermodulations present are located in the underdoped regime, where period-3 charge orders were observed in the bulk. Therefore, carrier concentration may not be essential to account for the aforementioned difference.

Given the magnetic properties of LCMO, we must certainly consider their effect on charge order. We found that $T_{\text {co }}$ is $45 \mathrm{~K}$ higher than $T_{\mathrm{m}}$, which marks the onset of macroscopic ferromagnetism in the LCMO layer. Ferromagnetism has been found to set in at the YBCO/LCMO interface slightly below $T_{\mathrm{m}}$ (ref. 32), in contrast to the temperature of $T_{\text {co }}$ that we observe. Therefore, the long-range ferromagnetism of LCMO does not appear to be directly relevant to the formation of the electronic supermodulation. While short-range ferromagnetism may possibly persist above $T_{\mathrm{m}}$, it is unlikely to play a major role in light of the abrupt onset at $T_{\mathrm{co}}$ and the lack of anomaly at $T_{\mathrm{m}}$ observed in the temperature evolution of the electronic supermodulation. In bulk YBCO, we note that the charge ordering onset is independent of external magnetic field. But magnetic field suppresses superconductivity and can consequently enhance the charge order near and below $T_{\mathrm{c}}$ (ref. 14). In the case of YBCO/LCMO, ferromagnetism at the interface or induced in the YBCO layer may exert a similar indirect effect on the electronic supermodulation via the exchange field. Whether the two can couple in a different way remains to be explored.

Therefore, we turn our attention to the effects of strain. Strain can affect the energetics of competing ordering tendencies or the forms in which a given tendency manifest by tipping the balance one way or the other. Compared with bulk YBCO, all thin films in our samples are subject to an additional (biaxial) tensile strain due to the $\mathrm{SrTiO}_{3}$ substrate ${ }^{33}$. This strain may undergo subtle variations between sample growth with and without the LCMO layer due to changes in the growth environment and relaxation within the LCMO layer. These considerations may help to rationalize why we could not find diffraction peaks associated with an electronic supermodulation (whether period-3 or period-4) on $30-\mathrm{nm}$ YBCO thin films $\left(T_{\mathrm{c}} \sim 60 \mathrm{~K}\right)$ grown directly on the $\mathrm{SrTiO}_{3}$ substrate. Incidentally, we note that no period-3 charge order has yet been reported on YBCO thin films, including ref. 13 in which charge orders were found in NBCO thin films but not in YBCO. A systematic study of YBCO thin films with varying substrates and post-annealing conditions may provide a good means to elucidate the effects of strain on the charge ordering phenomena in YBCO, as well as whether other effects brought about by the LCMO layer are also essential to the manifestation of period-4 electronic supermodulation.

The aforementioned three factors are implicated with another phenomenon for which YBCO/LCMO heterostructures have been known-the long-range proximity effect between superconductivity and ferromagnetism as the two major competing orders in the system. As in other superconductor-ferromagnet heterostructures $^{34}$, superconductivity in YBCO and ferromagnetism in LCMO tend to suppress each other at the interface and extend their influence into the opposing layer. But because of the short superconducting coherence length along the $c$ axis in YBCO ( $<3 \AA$ ), the high spin polarization (close to $100 \%$ ) and large exchange splitting $(\sim 3 \mathrm{eV})$ in LCMO, the mutual suppressions of both orders in this system are expected to be limited to within a close vicinity of the interface ( $\sim 1-\mathrm{nm}$ away). Instead, several 
experiments have uncovered persistent effects that exist over a remarkably long range of several tens of nanometres ${ }^{33,35-38}$. Understanding such an unusual effect requires consideration of factors beyond (singlet) superconductivity and ferromagnetism. While long-range effects caused by charge transfer ${ }^{39,40}$, $\operatorname{strain}^{33}$, disorder, defects ${ }^{41}$ and triplet superconductivity ${ }^{42,43}$ are under debate, a possible relevance of competing orders has been discussed very little, in part because existing candidates for the competing order, such as antiferromagnetism ${ }^{32}$ induced at the interface, are not sufficiently long ranged. Our observation of a 3D long-range order inherent in YBCO/LCMO heterostructures that competes with superconductivity provides an angle for addressing this problem: superconductivity far from the interface can be directly suppressed by the electronic supermodulation even if ferromagnetism is absent or limited to the interfacial region. To what degree this mechanism is relevant to the observed long-range proximity effect remains to be seen.

\section{Methods}

Samples. Each of the studied YBCO thin films has 25 u.c. and a thickness of $\sim 30 \mathrm{~nm}$, and was grown by pulsed laser deposition on top of a 25 u.c. $(\sim 10 \mathrm{~nm})$ LCMO layer, which was initially deposited on the (001)-oriented $\mathrm{SrTiO}_{3}$ (STO) substrate. $\operatorname{A~} \operatorname{KrF}(\lambda=248 \mathrm{~nm})$ excimer laser, with $10 \mathrm{~Hz}$ repetition rate and $250 \mathrm{~mJ}$ power, was used to evaporate the target. The STO substrate was treated with $\mathrm{HF}-\mathrm{NH}_{4} \mathrm{~F}$ buffer solution to produce a uniform $\mathrm{TiO}_{2}$ termination. Direct deposition of YBCO/LCMO on top of the $\mathrm{TiO}_{2}$-terminated STO makes a $\mathrm{MnO}_{2}$-terminated interface $\left(\mathrm{La}_{0.7} \mathrm{Ca}_{0.3}-\mathrm{MnO}_{2}-\mathrm{BaO}-\mathrm{CuO}_{2}\right)$ between $\mathrm{YBCO}$ and LCMO (sample \#2), whereas insertion of a buffer layer of 1.5 u.c. $\mathrm{SrRuO}_{3}$ (SRO) between the STO substrate and LCMO layer produces a $\mathrm{La}_{0.7} \mathrm{Ca}_{0.3} \mathrm{O}$-terminated interface $\left(\mathrm{MnO}_{2}-\mathrm{La}_{0.7} \mathrm{Ca}_{0.3}-\mathrm{CuO}_{2}-\mathrm{BaO}\right.$; sample \#1) (refs 31,44). The growth temperature was set at 700 and $715^{\circ} \mathrm{C}$ with an oxygen pressure of 80 and 200 mtorr for the deposition of LCMO and YBCO layers, respectively. All oxide layers were grown layer by layer as monitored in situ by reflection high-energy electron diffraction (Supplementary Fig. 1). An annealing treatment at $600{ }^{\circ} \mathrm{C}$ with an oxygen pressure of 700 torr was performed in situ on each grown sample for $40 \mathrm{~min}$ followed by a slow cool down to room temperature to ensure full oxygenation. A capping layer of $\sim 10-\mathrm{nm} \mathrm{LaAlO}_{3}$ was used to protect a portion of the sample surface for ex situ REXS measurements.

\section{Characterizations. Four-probe resistivity and SQUID magnetization} measurements were performed on the sample soon after the growth. The superconducting transition temperature $\left(T_{\mathrm{c}}\right)$ of samples \#1 and \#2 are $55 \pm 10$ and $72 \pm 7 \mathrm{~K}$, respectively. During the magnetization measurement, a 100 Oe magnetic field was applied perpendicular to the sample surface, and the field-cooled results are presented. The magnetic Curie temperature $\left(T_{\mathrm{m}}\right)$ was found to be the same for all samples studied, $\sim 175 \mathrm{~K}$, irrespective of slight variations in the growth and annealing conditions. This value is similar to that reported earlier on YBCO/LCMO in ref. 32.

REXS. REXS experiments were mainly carried out in the scattering endstation at BL 4.0.2 of the Advanced Light Source (ALS). Main results have been reproduced in experiments performed in two additional scattering endstations located at BL 8.0.1 of the ALS and the REIXS beamline of the Canadian Light Source. Scattered intensity was measured using an in-vacuum CCD (charge-coupled device) detector. CCD images were captured at a combination of detector and sample incidence angles, with each experimental geometry corresponding to a specified $(H, 0, L)$ reflection near the centre of the CCD screen. Within each raw CCD image, the horizontal axis is parallel to the primary scattering plane, cutting through the diffraction peak in the $(H, 0, L)$ plane with a trajectory depending on the photon energy, and the sample and detector angles. The vertical axis of each image is perpendicular to the scattering plane and essentially parallel to the $(0, K, 0)$ direction. Figure $1 \mathrm{c}$ shows one such image projected onto the $(H, K, 0)$ plane. Intensity line cuts, as a function of $H$ at fixed $K, L$ and photon energy (Figs $1 \mathrm{~d}-\mathrm{f}$ and $3 \mathrm{a}, \mathrm{c}$ ), as a function of $L$ at fixed $H, K$ and photon energy (Fig. 2a,b) and as a function of photon energy at fixed $H, K$ and $L$ (Fig. 4a), are extracted from a sequence of CCD images taken at different sample and detector angles with the centre of each image corresponding to the designated $(H, 0, L)$. We note that when the diffraction condition is met for the supermodulation peak, its intensity is only in or around the central area of the CCD. In addition, reciprocal space maps constructed from the full CCD pixel array were used to confirm the 3D nature of the supermodulation (Supplementary Fig. 4; Supplementary Note 1). In-plane lattice constant $a=3.855 \AA$ is assumed by averaging the $a$ and $b$ axis lattice constants reported on bulk and thin film samples at comparable doping levels ${ }^{13}$. The $c$ axis lattice constant $c=11.64 \AA$ is an averaged value of those determined from the $(0,0,1)$ (lattice) Bragg peak for samples at $\sim 50 \mathrm{~K}$. Various factors contributed to the estimated experimental uncertainties in the supermodulation wavevector, including variations in the measured lattice constant among different experiments and as a function of temperature, imperfect sample-to-beam alignment, crystalline mosaicity, curvature of the thin films and so on.

\section{References}

1. Fradkin, E., Kivelson, S. A. \& Tranquada, J. M. Theory of intertwined orders in high temperature superconductors. Rev. Mod. Phys. 87, 457-482 (2015).

2. Tranquada, J. M., Sternlieb, B. J., Axe, J. D., Nakamura, Y. \& Uchida, S. Evidence for stripe correlations of spins and holes in copper oxide superconductors. Nature 375, 561-563 (1995).

3. Fink, J. et al. Charge ordering in $\mathrm{La}_{1.8}{ }_{x} \mathrm{Eu}_{0.2} \mathrm{Sr}_{x} \mathrm{CuO}_{4}$ studied by resonant soft x-ray diffraction. Phys. Rev. B 79, 100502 (2009).

4. Abbamonte, P. et al. Spatially modulated 'Mottness' in $\mathrm{La}_{2}{ }_{x} \mathrm{Ba}_{x} \mathrm{CuO}_{4} . \mathrm{Nat}$. Phys. 1, 155-158 (2005).

5. Hanaguri, T. et al. A 'checkerboard' electronic crystal state in lightly hole-doped $\mathrm{Ca}_{2-x} \mathrm{Na}_{x} \mathrm{CuO}_{2} \mathrm{Cl}_{2}$. Nature 430, 1001-1005 (2004).

6. Hoffman, J. E. et al. A four unit cell periodic pattern of quasi-particle states surrounding vortex cores in $\mathrm{Bi}_{2} \mathrm{Sr}_{2} \mathrm{CaCu}_{2} \mathrm{O}_{8}+\delta$. Science 295, 466-469 (2002).

7. Howald, C., Eisaki, H., Kaneko, N. \& Kapitulnik, A. Coexistence of periodic modulation of quasiparticle states and superconductivity in $\mathrm{Bi}_{2} \mathrm{Sr}_{2} \mathrm{CaCu}_{2} \mathrm{O}_{8}+\delta$. Proc. Natl Acad. Sci. USA 100, 9705-9709 (2003).

8. da Silva Neto, E. H. et al. Ubiquitous interplay between charge ordering and high-temperature superconductivity in cuprates. Science 343, 393-396 (2014).

9. Wu, T. et al. Magnetic-field-induced charge-stripe order in the hightemperature superconductor $\mathrm{YBa}_{2} \mathrm{Cu}_{3} \mathrm{O}_{y}$. Nature 477, 191-194 (2011).

10. Comin, R. et al. Charge order driven by Fermi-arc instability in $\mathrm{Bi}_{2} \mathrm{Sr}_{2 x} \mathrm{La}_{x} \mathrm{CuO}_{6+\delta}$. Science 343, 390-392 (2014).

11. da Silva Neto, E. H. et al. Charge ordering in the electron-doped superconductor $\mathrm{Nd}_{2 x} \mathrm{Ce}_{x} \mathrm{CuO}_{4}$. Science 347, 282-285 (2015).

12. Wise, W. D. et al. Charge-density-wave origin of cuprate checkerboard visualized by scanning tunnelling microscopy. Nat. Phys. 4, 696-699 (2008).

13. Ghiringhelli, G. et al. Long-range incommensurate charge fluctuations in ( $\mathrm{Y}, \mathrm{Nd}) \mathrm{Ba}_{2} \mathrm{Cu}_{3} \mathrm{O}_{6+x}$. Science 337, 821-825 (2012).

14. Chang, J. et al. Direct observation of competition between superconductivity and charge density wave order in $\mathrm{YBa}_{2} \mathrm{Cu}_{3} \mathrm{O}_{6.67}$. Nat. Phys. 8, 871-876 (2012)

15. Achkar, A. J. et al. Distinct charge orders in the planes and chains of ortho-III-ordered $\mathrm{YBa}_{2} \mathrm{Cu}_{3} \mathrm{O}_{6+\delta}$ superconductors identified by resonant elastic X-ray scattering. Phys. Rev. Lett. 109, 167001 (2012).

16. Blanco-Canosa, S. et al. Resonant X-ray scattering study of charge-density wave correlations in $\mathrm{YBa}_{2} \mathrm{Cu}_{3} \mathrm{O}_{6+x}$. Phys. Rev. B 90, 054513 (2014).

17. Hucker, M. et al. Competing charge, spin, and superconducting orders in underdoped $\mathrm{YBa}_{2} \mathrm{Cu}_{3} \mathrm{O}_{y}$. Phys. Rev. B 90, 054514 (2014).

18. Tabis, W. et al. Charge order and its connection with Fermi-liquid charge transport in a pristine high- $T_{c}$ cuprate. Nat. Commun. 5, 5875 (2014).

19. Wu, T. et al. Incipient charge order observed by NMR in the normal state of $\mathrm{YBa}_{2} \mathrm{Cu}_{3} \mathrm{O}_{y}$. Nat. Commun. 6, 6438 (2015).

20. Nie, L., Tarjus, G. \& Kivelson, S. A. Quenched disorder and vestigial nematicity in the pseudogap regime of the cuprates. Proc. Natl Acad. Sci. USA 111, 79807985 (2014).

21. Thampy, V. et al. Comparison of charge modulations in $\mathrm{La}_{1.875} \mathrm{Ba}_{0.125} \mathrm{CuO}_{4}$ and $\mathrm{YBa}_{2} \mathrm{Cu}_{3} \mathrm{O}_{6.6}$. Phys. Rev. B 88, 024505 (2013).

22. Wiesenmayer, E. et al. Microscopic coexistence of superconductivity and magnetism in $\mathrm{Ba}_{1 x} \mathrm{~K}_{x} \mathrm{Fe}_{2} \mathrm{As}_{2}$. Phys. Rev. Lett. 107, 237001 (2011).

23. Hashimoto, M. et al. Direct spectroscopic evidence for phase competition between the pseudogap and superconductivity in $\mathrm{Bi}_{2} \mathrm{Sr}_{2} \mathrm{CaCu}_{2} \mathrm{O}_{8+\delta}$. Nat. Mater. 14, 37-42 (2015).

24. Islam, Z. et al. Four-unit-cell superstructure in the optimally doped $\mathrm{YBa}_{2} \mathrm{Cu}_{3} \mathrm{O}_{6.92}$ superconductor. Phys. Rev. Lett. 93, 157008 (2004).

25. Strempfer, J. et al. Oxygen superstructures throughout the phase diagram of (Y,Ca) $\mathrm{Ba}_{2} \mathrm{Cu}_{3} \mathrm{O}_{6+x}$. Phys. Rev. Lett. 93, 157007 (2004).

26. $\mathrm{Wu}, \mathrm{H} . \mathrm{-H}$. et al. Charge stripe order near the surface of 12-percent doped $\mathrm{La}_{2}{ }_{x} \mathrm{Sr}_{x} \mathrm{CuO}_{4}$. Nat. Commun. 3, 1023 (2012).

27. Hawthorn, D. G. et al. Resonant elastic soft $\mathrm{x}$-ray scattering in oxygen-ordered $\mathrm{YBa}_{2} \mathrm{Cu}_{3} \mathrm{O}_{6+\delta}$. Phys. Rev. B 84, 075125 (2011).

28. Salluzzo, M. et al. Thickness effect on the structure and superconductivity of $\mathrm{Nd}_{1.2} \mathrm{Ba}_{1.8} \mathrm{Cu}_{3} \mathrm{O}_{z}$ epitaxial films. Phys. Rev. B 72, 134521 (2005).

29. Le Tacon, M. et al. Inelastic X-ray scattering in $\mathrm{YBa}_{2} \mathrm{Cu}_{3} \mathrm{O}_{6.6}$ reveals giant phonon anomalies and elastic central peak due to charge-density-wave formation. Nat. Phys. 10, 52-58 (2013).

30. Chakhalian, J. et al. Orbital reconstruction and covalent bonding at an oxide interface. Science 318, 1114-1117 (2007).

31. Lin, J. Y., Tra, V. T. \& Chu, Y. H. Charge transfer at $\mathrm{YBa}_{2} \mathrm{Cu}_{3} \mathrm{O}_{7} /$ $\mathrm{La}_{0.7} \mathrm{Ca}_{0.3} \mathrm{MnO}_{3}$ interface. Bull. Am. Phys. Soc. 59, S6.2 (2014).

32. Chakhalian, J. et al. Magnetism at the interface between ferromagnetic and superconducting oxides. Nat. Phys. 2, 244-248 (2006). 
33. Hoppler, J. et al. Giant superconductivity-induced modulation of the ferromagnetic magnetization in a cuprate manganite superlattice. Nat. Mater. 8, 315-319 (2009).

34. Buzdin, A. Proximity effects in superconductor-ferromagnet heterostructures Rev. Mod. Phys. 77, 935-976 (2005).

35. Sefrioui, Z. et al. Ferromagnetic/superconducting proximity effect in $\mathrm{La}_{0.7} \mathrm{Ca}_{0.3} \mathrm{MnO}_{3} / \mathrm{YBa}_{2} \mathrm{Cu}_{3} \mathrm{O}_{7-\delta}$ superlattices. Phys. Rev. B 67, 214511 (2003).

36. Holden, T. et al. Proximity induced metal-insulator transition in $\mathrm{YBa}_{2} \mathrm{Cu}_{3} \mathrm{O}_{7} / \mathrm{La}_{0.7} \mathrm{Ca}_{0.3} \mathrm{MnO}_{3}$ superlattices. Phys. Rev. B 69, 064505 (2004).

37. Pena, V. et al. Coupling of superconductors through a half-metallic ferromagnet: Evidence for a long-range proximity effect. Phys. Rev. B 69, 224502 (2004)

38. Driza, N. et al. Long-range transfer of electron-phonon coupling in oxide superlattices. Nat. Mater. 11, 675-681 (2012).

39. Hoffmann, A. et al. Suppressed magnetization in $\mathrm{La}_{0.7} \mathrm{Ca}_{0.3} \mathrm{MnO}_{3} /$ $\mathrm{YBa}_{2} \mathrm{Cu}_{3} \mathrm{O}_{7-\delta}$ superlattices. Phys. Rev. B 72, 140407(R) (2005).

40. Chien, T. Y. et al. Visualizing short-range charge transfer at the interfaces between ferromagnetic and superconducting oxides. Nat. Commun. 4, 2336 (2013).

41. Zhang, H., Gauquelin, N., Botton, G. A. \& Wei, J. Y. T. Attenuation of superconductivity in manganite/cuprate heterostructures by epitaxially-induced CuO intergrowths. App. Phys. Lett. 103, 052606 (2013).

42. Kalcheim, Y., Kirzhner, T., Koren, G. \& Millo, O. Long-range proximity effect in $\mathrm{La}_{0.7} \mathrm{Ca}_{0.3} \mathrm{MnO}_{3} / \mathrm{YBa}_{2} \mathrm{Cu}_{3} \mathrm{O}_{7-\delta}$ ferromagnet/superconductor bilayers: evidence for induced triplet superconductivity in the ferromagnet. Phys. Rev. B 83, 064510 (2011)

43. Visani, C. et al. Equal-spin Andreev reflection and long-range coherent transport in high-temperature superconductor/half-metallic ferromagnet junctions. Nat. Phys. 8, 539-543 (2012).

44. Yu, P. et al. Interface control of bulk ferroelectric polarization. Proc. Natl Acad. Sci. USA 109, 9710-9715 (2012).

\section{Acknowledgements}

We thank M. Hashimoto for useful discussion. The work at Boston College was supported by a BC startup fund (J.H. and R.-H.H.), US NSF CAREER award DMR-1454926 (R.-H.H., in part), NSF Graduate Research Fellowship DGE-1258923 (T.R.M.) and NSF MRI grant DMR-1337576 (M.J.G.). The work in NCTU is supported by Ministry of Science and Technology, R.O.C. (MOST 103-2119-M-009-003-MY3), Center for Interdisciplinary Science of National Chiao Tung University, Ministry of Education, Taiwan (MOE-ATU 101W961). Research was mainly performed at the ALS, which is supported by the Director, Office of Science, Office of Basic Energy Sciences, of US DOE under contract no. DE-AC02-05CH11231. Research was partially performed at the Canadian Light Source (proposal \#19-5803), which is funded by the CFI, NSERC, NRC, CIHR, the Government of Saskatchewan, WD Canada, and the University of Saskatchewan.

\section{Author contributions}

J.H., P.S. and T.R.M. led the REXS experiments with R.-H.H. and Q.H.; V.T.T., J.-Y.L., and Y.-H.C. grew and characterized the samples. P.S., Y.-D.C., W.L.Y. and E.A. maintained the REXS endstations. J.H., P.S. and R.-H.H. analysed the data with assistance from J.K.; J.H., P.S. and R.-H.H. wrote the paper with suggestions and comments by E.A. and T.R.M.; R.-H.H., E.A., Y.-H.C., J.-Y.L. and M.J.G. were responsible for project direction, planning and infrastructure.

\section{Additional information}

Supplementary Information accompanies this paper at http://www.nature.com/ naturecommunications

Competing financial interests: The authors declare no competing financial interests.

Reprints and permission information is available online at http://npg.nature.com/ reprintsandpermissions/

How to cite this article: $\mathrm{He}$, J. et al. Observation of a three-dimensional quasi-long-range electronic supermodulation in $\mathrm{YBa}_{2} \mathrm{Cu}_{3} \mathrm{O}_{7-x} / \mathrm{La}_{0.7} \mathrm{Ca}_{0.3} \mathrm{MnO}_{3}$ heterostructures. Nat. Commun. 7:10852 doi: 10.1038/ncomms10852 (2016).

(c) (i) This work is licensed under a Creative Commons Attribution 4.0 International License. The images or other third party material in this article are included in the article's Creative Commons license, unless indicated otherwise in the credit line; if the material is not included under the Creative Commons license, users will need to obtain permission from the license holder to reproduce the material. To view a copy of this license, visit http://creativecommons.org/licenses/by/4.0/ 